\title{
La carte sanitaire de la ville de Lubumbashi, République Démocratique du Congo Partie II : analyse des activités opérationnelles des structures de soins
}

\author{
Mukalenge Chenge ${ }^{1}$, Jean Van der Vennet ${ }^{2}$, Denis Porignon ${ }^{3,4}$, \\ Numbi Luboya ${ }^{1}$, Ilunga Kabyla ${ }^{1}$ et Bart Criel $^{2}$
}

\begin{abstract}
Résumé: Les structures de santé dans la ville de Lubumbashi tentent d'offrir à la population des soins de santé en réponse à ses besoins ressentis. Cet article présente et analyse la nature et le volume des soins offerts par les différents prestataires institutionnels aux différents niveaux du système de santé pour l'ensemble de l'année 2006. Pratiquement toutes les structures offrent des soins curatifs. Le taux d'utilisation moyen pour l'ensemble de la ville est de 0,37 nouveaux cas par habitant par an. La population utilise plus souvent l'hôpital que les structures intermédiaires et celles de première ligne. Des maternités sont disponibles dans près de $2 / 3$ des structures de première ligne, dans toutes les structures intermédiaires et dans tous les hôpitaux. Neuf accouchements sur dix sont assistés avec un taux de césarienne de moins de $2 \%$. La vaccination est réalisée dans $2 / 3$ des structures de première ligne avec un taux de couverture de la $3^{\text {ème }}$ dose du vaccin trivalent contre la diphtérie, le tetanos et la coqueluche (DTC3) de $49 \%$. Seulement quelques structures de première ligne dépistent les cas de tuberculose pulmonaire à microscopie positive avec un taux de dépistage de $44 \%$. La présente étude, basée sur une mesure quantitative, met en évidence une discordance entre l'offre et la demande. Elle contribue à une meilleure compréhension de l'offre de soins à Lubumbashi même si les questions de la qualité des soins et de l'accessibilité financière aux soins restent encore en suspens. (Global Health Promotion, 2010; 17(3) : pp. 75-84)
\end{abstract}

Mots clés: Lubumbashi, paquet d'activités, RD du Congo, services de santé urbains, utilisation des services de santé

\section{Introduction}

Cet article prolonge l'étude sur la carte sanitaire de la ville de Lubumbashi. La première partie qui a traité de la problématique de la couverture sanitaire a inventorié les structures formelles de soins existantes, étudié l'évolution de leur apparition dans le temps, déterminé leur répartition géographique et identifié les différents prestataires institutionnels (gouvernement, églises, ONG locales, entreprises et privés indépendants) et opérationnels qui y travaillent (1).
Depuis son adhésion à la stratégie des soins de santé primaires et sa souscription en 1980 à la charte africaine de développement sanitaire, la République Démocratique du Congo (RD Congo) a toujours défini sa politique sanitaire en partant des principes de base d'organisation et de fonctionnement des services de santé à finalité publique (2). Cependant, suite à la crise multisectorielle que connaît le pays depuis le début des années 1990, le secteur de la santé est confronté à plusieurs problèmes. Il s'agit notamment de la désintégration du

1. Correspondance à : Mukalenge Chenge, École de Santé Publique de l'Université de Lubumbashi, RD du Congo (chengefaust@yahoo.fr).

2. Département de Santé Publique de l'Institut de Médecine Tropicale Prince Léopold, Belgique.

3. Ecole de Santé Publique de l'Université Libre de Bruxelles, Belgique.

4. Department for Health System Governance and Service Delivery, WHO, Genève, Suisse.

(Cet article a été soumis le $1^{\text {er }}$ aout 2009. Après évaluation par des pairs, il a été accepté pour publication le 15 mars 2010)

Global Health Promotion 1757-9759; Vol 17(3): 75-84; 375174 Copyright () The Author(s) 2010, Reprints and permissions: http://www.sagepub.co.uk/journalsPermissions.nav DOI: 10.1177/1757975910375174 http://ghp.sagepub.com 
système avec une marginalisation de l'hôpital et le développement des stratégies de survie, tant sur le plan individuel qu'institutionnel, caractérisé entre autres par la prolifération " anarchique » des structures de soins (2).

Devant ce sombre tableau, une réforme du secteur de la santé en RD Congo s'impose. C'est ainsi que le Ministère de la Santé a élaboré la Stratégie de Renforcement du Système de Santé (SRSS) dont l'un des axes principaux est le développement / la revitalisation des zones de santé et la correction des discor-dances faites à ce niveau.

Le développement ou la revitalisation des zones de santé prônée par la SRSS (2) requiert une bonne connaissance des activités opérationnelles de soins de santé réalisées dans les structures de soins existantes. Ceci est plus important en milieu urbain où les systèmes de santé sont caractérisés par une grande diversité de prestataires offrant une multiplicité de services $(1,3-5)$.

Selon l'enquête menée par l'observatoire du changement urbain de Lubumbashi (6), le taux de morbidité générale est de $18,5 \%$ à Lubumbashi avec un taux de mortalité de $1,6 \%$. La même enquête indique que la morbidité à Lubumbashi est dominée par des pathologies parasitaires et infectieuses, le paludisme arrive en tête au niveau communautaire (67\% des cas de maladie) comme dans les structures de soins (plus de $20 \%$ des cas reçus). L'anémie (souvent due au paludisme) est la cause la plus fréquente de la mortalité, surtout chez les enfants de moins de 5 ans, tandis que les complications liées à la grossesse sont la cause la plus fréquente de la mortalité maternelle à Lubumbashi $(6,7)$.

La présente étude analyse de manière critique les principales interventions mises en place pour soulager la souffrance de la population dans les structures de soins de Lubumbashi. De façon spécifique, il s'agit de déterminer l'offre et le niveau de la demande de soins dans la ville et de comparer le volume d'activités de différents prestataires.

Les résultats de cette étude rentrent dans le cadre de l'élaboration de la carte sanitaire de la ville et permettent une meilleure connaissance de la réalité du terrain. Ils ont été présentés aux autorités sanitaires nationales et provinciales et ils devraient servir d'instrument de négociation et de planification d'une offre de soins rationnelle (8-10) et adaptée au contexte.

\section{Contexte}

La ville de Lubumbashi, sise au Sud-Est de la province du Katanga, en est le chef-lieu. Elle s'étend sur $747 \mathrm{~km}^{2}$ dont $140 \mathrm{~km}^{2}$ urbanisés. Fin 2006, sa population est estimée à 1500000 habitants dont environ 1400000 habitent dans la partie urbanisée (soit une densité de 10000 habitants $/ \mathrm{km}^{2 \mathrm{i}}$ ). C'est la deuxième ville de la RD Congo après Kinshasa, la capitale.

En 2006, la ville de Lubumbashi comptait 251 structures de soins réparties comme suit(1):

- 219 structures de première ligne, c'est-à-dire offrant uniquement des soins ambulatoires et des accouchements par voie basse;

- 23 structures intermédiaires, c'est-à-dire pratiquant de la chirurgie générale majeure (césariennes, appendicectomies, cures de hernie ainsi que différentes laparotomies pour grossesse ectopique, myomes...) et des hospitalisations, mais dont la capacité d'accueil effective ( $\leq 50$ lits) et/ou le plateau technique sont trop faibles pour qu'elles soient considérées comme des hôpitaux;

- 9 établissements hospitaliers, c'est-à-dire organisant au moins des services de chirurgie, pédiatrie, médecine générale et gynécologie- obstétrique et dont le nombre de lits effectifs dépasse 50 ; qu'ils aient ou non toute la gamme d'unités d'appui tels que l'imagerie, le laboratoire, la pharmacie.

Ces deux dernières catégories de structures disposent toutes de services ambulatoires.

\section{Méthodologie}

La collecte des informations sur le volume d'activités de chaque structure de soins a été une étape laborieuse dans l'élaboration de la carte sanitaire de la ville de Lubumbashi. En effet, les rapports SNIS (Système National d'Information Sanitaire) qui auraient dû servir de base à la collecte de données de prestation des structures sont souvent incomplets. Ils ne reprennent en effet que des données des structures engagées dans une collaboration explicite avec le Bureau Central de la Zone de Santé (BCZS) qui fait office d'équipe cadre de district en RD Congo.

Par souci de complétude des informations et d'exhaustivité des cas, nous avons collecté les informations pour toute l'année 2006 dans chaque structure de soins à l'aide d'une grille de collecte de 
données. Les sources d'information utilisées sont les rapports d'activités et les registres de consultation (11). La collecte des données a été faite par 11 infirmiers superviseurs de chacune de 11 zones de santé de Lubumbashi. Ces infirmiers ont été sélectionnés sur la base d'une bonne connaissance de leurs zones, de la familiarité dont ils jouissent auprès des prestataires et de leur expérience dans le remplissage de ce genre de grille. Ils ont ensuite été formés pendant une journée sur la manière de rechercher et de notifier les informations requises sur le terrain. La collecte de données proprement dite a eu lieu au cours du premier trimestre 2007 et le contrôle de qualité a été assuré par le premier auteur. Dans les structures de première ligne, les informations recueillies ont porté sur le nombre de nouveaux cas de consultations curatives, le nombre d'accouchements par voie basse, le nombre d'enfants ayant reçu leur $3^{\text {ème }}$ dose du vaccin DTC et le nombre de tuberculeux pulmonaires à microscopie positive diagnostiqués. Dans les structures intermédiaires et les hôpitaux, ces informations concernaient le nombre de malades hospitalisés et sortis, le nombre de journées d'hospitalisation, le nombre d'accouche-ments par voie basse, le nombre de césariennes, le nombre d'autres interventions relevant par convention de la chirurgie générale majeure et le nombre de consultations médicales ${ }^{\text {ii }}$.

Le choix de collecter les données relatives à ces activités a été motivé par le souci de respecter la spécificité du paquet d'activités à chaque niveau de soins.
Les consultations curatives et les accouchements sont considérés comme des activités communes aux structures de première ligne et aux hôpitaux, même si l'on sait par ailleurs que les hôpitaux, sans en avoir la vocation, participent aussi aux autres activités retenues ici comme « propres » à la première ligne $(12,13)$.

La plupart des données que nous avons recueillies ne sont pas distribuées de façon gaussienne (12). Pour cette raison, nous analysons les données relatives aux consultations curatives, aux accouchements, à la vaccination, au diagnostic de la tuberculose et à la capacité d'accueil en utilisant la médiane, le quartile inférieur (QI) et le quartile supérieur (QS) ainsi que l'intervalle de confiance à $95 \%$ (IC à $95 \%$ ). Pour la comparaison du volume d'activités des niveaux et types de structures de soins, la différence observée entre deux médianes est statistiquement significative si les deux intervalles de confiance ne se chevauchent pas. La comparaison du volume d'activités entre les niveaux et types de structures de soins permet d'identifier les principaux pôles d'attraction ou de recours des patients.

Nous avons comparé les structures intermédiaires et les hôpitaux en utilisant la durée moyenne de séjour (DMS) et le taux d'occupation moyen de lits (TOM) (14). D'autres indicateurs (ratio de nouveaux cas de consultation curative par habitant par an, taux d'accouchements assistés, taux de césariennes, taux de couverture vaccinale et taux de dépistage de TPM+) sont calculés pour évaluer le niveau d'utilisation des services de santé offerts. Nous donnons dans le Tableau 1 les valeurs de

Tableau 1. Valeurs de référence nationales des indicateurs et proxy utilisés dans cette étude

\begin{tabular}{|c|c|c|c|}
\hline Activités & Indicateurs/proxy & Valeurs de référence & Sources de la valeur de référence \\
\hline Consultations curatives & Taux d'utilisation & $\geq 0,5 \mathrm{NC} /$ habitant/an & $\begin{array}{l}\text { Ministère de la santé : Normes et } \\
\text { directives de la zone de santé, } 2003\end{array}$ \\
\hline \multirow[t]{2}{*}{ Accouchements } & $\begin{array}{l}\text { Taux d'accouchements } \\
\text { assistés par an }\end{array}$ & $\geq 80 \%$ & $\begin{array}{l}\text { PNSR* : Normes et directives de la } \\
\text { santé de la reproduction, } 2008\end{array}$ \\
\hline & Taux brut de natalité & $4 \%$ & $\begin{array}{l}\text { Enquête Démographique et } \\
\text { Sanitaire de } 2007\end{array}$ \\
\hline \multirow[t]{2}{*}{ Vaccinations } & $\begin{array}{l}\text { Taux de couverture } \\
\text { en DTC3 }\end{array}$ & $\geq 90 \%$ & $\mathrm{PEV}^{* *}$ : Plan d'action 2009 \\
\hline & Population cible & $4 \%$ & \\
\hline $\begin{array}{l}\text { Dépistage/diagnostic } \\
\text { de la tuberculose }\end{array}$ & $\begin{array}{l}\text { Taux de détection de } \\
\text { TPM+ } \\
\text { Incidence TPM+ }\end{array}$ & $\begin{array}{l}\geq 70 \% \\
\geq 150 \text { cas } / 100000 \\
\text { habitants }\end{array}$ & $\begin{array}{l}\text { PNT }^{* * *} \text { : Guide de prise en charge } \\
\text { de la tuberculose } 2008\end{array}$ \\
\hline
\end{tabular}

* Programme national de la santé de la reproduction ; * Programme élargi de vaccination ; ** Programme national de lutte contre la tuberculose. 
Tableau 2. Consultations curatives : contribution et volume de travail aux différents niveaux de soins, en 2006

\begin{tabular}{lccc}
\hline & $\begin{array}{c}\text { Nombre de structures } \\
\text { qui font des consultations } \\
\text { curatives à chaque niveau } \\
\text { de soins (\%) }\end{array}$ & $\begin{array}{c}\text { Nombre de NC de } \\
\text { consultations curatives } \\
(\% \text { du total) }\end{array}$ & $\begin{array}{c}\text { Nombre médian de } \\
\text { consultations curatives par } \\
\text { jour ouvrable par niveau } \\
\text { Niveaux de soins [ICà 95\%] }\end{array}$ \\
\hline $\begin{array}{l}\text { Structures de soins de } \\
\text { 1ierre ligne }\end{array}$ & $210 / 219(959)$ & $313674(56)$ & $4,7[4,2-5,2]$ \\
Structures intermédiaires & $23 / 23(100)$ & $64229(11)$ & $6,6[3,8-9,4]$ \\
Hôpitaux & $9 / 9(100)$ & $183928(33)$ & $69,2[33,9-104,5]$ \\
TOTAL & - & $561831(100)$ & - \\
\hline
\end{tabular}

référence (des objectifs nationaux) de ces indicateurs et les proxys ayant servi de base de calcul pour appréhender les déficits dans l'utilisation des services.

Les logiciels Excel et SPSS (Statistical Package for Social Sciences) ont servi aux calculs de ces différents indicateurs. Les résultats sont présentés et analysés en utilisant les trois niveaux de structuration opérationnelle (structures de première ligne, structures intermédiaires et hôpitaux) et la même nomenclature des prestataires institutionnels (gouvernement, Église catholique, autres Églises, ONG locales, entreprises, privés indépendants) adoptés pour les services de santé de la ville de Lubumbashi (1).

\section{Présentation et analyse des activités des structures de soins}

Nous présentons et analysons d'abord les activités communes aux structures de $1^{\text {ère }}$ ligne, aux structures intermédiaires et aux hôpitaux. Les activités « propres » à chacun des 3 niveaux sont présentées et analysées ensuite.

Si les structures intermédiaires et hospitalières offrent l'ensemble des activités de soins, il n'en est pas ainsi des structures de première ligne. En effet, les tableaux 2-7 indiquent chaque fois dans quelle proportion les différents prestataires de la première ligne de soins ont intégré dans leurs structures les activités retenues dans le cadre de cette étude comme étant spécifiques de la première ligne.

\section{Activités communes aux 3 types de structures}

\section{Consultations curatives}

En l'absence d'un système d'orientation-recours formellement organisé, nous considérons les consultations réalisées dans les structures intermédiaires et dans les hôpitaux comme des consultations de premier contact. Ainsi, le Tableau 2 montre qu'au cours de l'année 2006, pour l'ensemble des structures de soins on a enregistré 561831 nouvelles consultations curatives. Le taux d'utilisation global de services curatifs pour les 3 types de structures confondus est donc de 0,37 NC/habitant/an (561 831/1 500 000).

Le Tableau 2 permet aussi de comparer le volume de prestations entre les trois niveaux de soins, c'està-dire le nombre médian de consultations réalisées par jour ouvrable (275 jours ouvrables par an) et par structure : nous remarquons que les différences ainsi observées ne sont pas statistiquement significatives entre première ligne et niveau intermédiaire ; mais le sont lorsqu'on compare chacun des deux premiers niveaux avec l'hôpital.

Le même raisonnement appliqué aux prestataires de la première ligne (Tableau 3 ) montre qu'en dépit de leur contribution relativement faible $(5 \%)$ à l'ensemble du volume des consultations réalisées à la première ligne, les structures de soins des entreprises ont le volume de travail le plus important avec une médiane de 27,4 consultations par structure par jour ouvrable $(\mathrm{QI}=27,2 ; \mathrm{QS}=27,6)$ et c'est statistiquement significatif. Les différences notées entre les autres prestataires pris deux à deux ne sont pas statistiquement significatives.

\section{Accouchements}

Pour des raisons de cohérence, nous présentons dans cette partie les résultats des accouchements par voie basse qui sont réalisés à tous les niveaux de soins, aussi bien que ceux des césariennes qui sont effectuées seulement dans les structures intermédiaires et les hôpitaux.

Le Tableau 4 indique qu'au total 52123 accouchements ont été enregistrés au cours de l'année 2006 
Tableau 3. Consultations curatives : contribution et volume de travail dans les structures de $1^{\text {ère }}$ ligne réparties selon leur appartenance institutionnelle, en 2006

\begin{tabular}{lccc}
\hline $\begin{array}{l}\text { Appartenance } \\
\text { institutionnelle }\end{array}$ & $\begin{array}{c}\text { Nombre de structures qui } \\
\text { font des consultations } \\
\text { curatives }(\%)\end{array}$ & $\begin{array}{c}\text { Nombre de consultations } \\
\text { curatives en 2006 } \\
\text { \% du total) }\end{array}$ & $\begin{array}{c}\text { Nombre médian de consultations } \\
\text { curatives par jour ouvrable par } \\
\text { structure [IC à 95\%] }\end{array}$ \\
\hline Gouvernement & $15 / 15(100)$ & $26067(8)$ & $2,5[0,3-4,7]$ \\
Église catholique & $17 / 23(73,9)$ & $47832(15)$ & $7,2[2,8-11,6]$ \\
Autres Églises & $7 / 8(87,5)$ & $11616(4)$ & $4,4[2,4-6,4]$ \\
ONG & $5 / 5(100)$ & $3493(1)$ & $1,9[-0,1-4,0)]$ \\
Entreprises & $2 / 4(50)$ & $15093(5)$ & $27,4[27,0-27,9]$ \\
Indépendants & $164 / 164(100)$ & $209573(67)$ & $4,5[4,0-4,9]$ \\
TOTAL & $210 / 219(95,9)$ & $313674(100)$ & - \\
\hline
\end{tabular}

Tableau 4. Nombre et pourcentage d'accouchements par niveau de soins, en 2006

\begin{tabular}{lcccc}
\hline & $\begin{array}{c}\text { Nombre de structures } \\
\text { qui font des } \\
\text { accouchements }(\%)\end{array}$ & $\begin{array}{c}\text { Nombre d'accouchements } \\
\text { par voie basse } \\
\text { (\% du total) }\end{array}$ & $\begin{array}{c}\text { Nombre de } \\
\text { césariennes } \\
\text { (\% du total) })\end{array}$ & $\begin{array}{c}\text { Nombre de } \\
\text { césariennes } / 100 \\
\text { accouchements }\end{array}$ \\
\hline Structures de 1 $1^{\text {ère }}$ ligne & $141 / 219(64,4)$ & $25093(49)$ & - & - \\
Structures intermédiaires & $23 / 23(100)$ & $8832(17)$ & $251(27)$ & 2,8 \\
Hôpitaux & $9 / 9(100)$ & $17251(34)$ & $696(73)$ & 3,9 \\
TOTAL & $\mathbf{1 7 3 / 2 5 1 ( 6 9 )}$ & $51176(100)$ & $\mathbf{9 4 7}(100)$ & $\mathbf{1 , 8}$ \\
\hline
\end{tabular}

dont 51176 par voie basse $(98,2 \%)$ et 947 par césarienne (soit un taux de césarienne de $1,8 \%$ ). Près de la moitié $(49 \%)$ de l'ensemble des accouchements assistés ont lieu dans les structures de première ligne.

Connaissant le nombre d'accouchements attendus $(60000)$, calculé à partir d'un taux de natalité de $4 \%$ (Tableau 1 ), on estime à $86,9 \%$ le taux d'accouchements assistés. On note en outre que plus de $25 \%$ des césariennes se déroulent dans des structures intermédiaires et que, par rapport aux accouchements qui y ont lieu, le taux de césariennes est $2,8 \%$ alors qu'il est de $3.9 \%$ dans les hôpitaux.

Le Tableau 5 renseigne que $64,4 \%$ des structures de première ligne - toutes appartenances confondues font des accouchements. La comparaison des structures en fonction du nombre d'accouchement réalisés montre qu'il n'y a pas de différence statistiquement significative entre les types de structures.

Tableau 5. Accouchements dans les structures de première ligne : contribution et volume de travail dans les structures de $1^{\text {ère }}$ ligne réparties selon leur appartenance institutionnelle, en 2006

\begin{tabular}{lccc}
\hline $\begin{array}{l}\text { Appartenance } \\
\text { institutionnelle }\end{array}$ & $\begin{array}{c}\text { Nombre de structures } \\
\text { qui font des } \\
\text { accouchements }(\%)\end{array}$ & $\begin{array}{c}\text { Nombre d'accouchements } \\
\text { réalisés }(\% \text { du total) }\end{array}$ & $\begin{array}{c}\text { Nombre médian } \\
\text { d'accouchements par mois } \\
\text { par structure [IC à 95\%] }\end{array}$ \\
\hline Gouvernement & $14 / 15(93,3)$ & $2526(10,1)$ & $6,75[-1,1-14,6]$ \\
Eglise catholique & $10 / 23(43,5)$ & $3425(13,6)$ & $16,3[4,2-28,3]$ \\
Autres Églises & $7 / 8(87,5)$ & $1455(5,8)$ & $12,4[2,1-22,7]$ \\
ONG & $3 / 5(60)$ & $992(4)$ & $37,9[6,7-69,2]$ \\
Entreprises & $0 / 4(0)$ & - & - \\
Indépendants & $107 / 164(65,2)$ & $16695(66,5)$ & $8[6,3-9,7]$ \\
TOTAL & $141 / 219(64,4)$ & $25093(100)$ & - \\
\hline
\end{tabular}


Tableau 6. Répartition des enfants ayant reçu leur $3^{\text {ème }}$ dose de DTC dans des structures de $1^{\text {èr }}$ ligne groupées selon l'appartenance institutionnelle et évaluation du volume de travail par type de structure, en 2006

\begin{tabular}{lccc}
\hline $\begin{array}{l}\text { Appartenance } \\
\text { institutionnelle }\end{array}$ & $\begin{array}{c}\text { Nombre de structures } \\
\text { qui vaccinent }(\%)\end{array}$ & $\begin{array}{c}\text { Nombre total de doses } \\
\text { de DTC3 administrées } \\
(\% \text { du total) }\end{array}$ & $\begin{array}{c}\text { Nombre médian de doses de } \\
\text { DTC3 administrées par mois } \\
\text { par structure [IC à 95\%] }\end{array}$ \\
\hline Gouvernement & $14 / 15(93,3)$ & $4658(16)$ & $19,9[11,4-28,4]$ \\
Eglise catholique & $20 / 23(87)$ & $6499(22)$ & $17,4[12,1-22,7]$ \\
Autres Églises & $7 / 8(87,5)$ & $1486(5)$ & $14,7[9,0-20,3]$ \\
ONG & $2 / 5(40)$ & $427(1)$ & $17,8[6,3-29,2]$ \\
Entreprises & $2 / 4(50)$ & $312(1)$ & $13,0[10,1-15,9]$ \\
Indépendants & $103 / 164(62,8)$ & $15988(55)$ & $9,4[7,7-11,2]$ \\
TOTAL & $\mathbf{1 4 8 / 2 1 9 ( 6 7 , 6 )}$ & $29370(100)$ & - \\
\hline
\end{tabular}

Tableau 7. Diagnostic de la tuberculose pulmonaire à microscopie positive dans les structures de première ligne groupées selon l'appartenance institutionnelle et évaluation du volume de travail par type de structure, 2006

\begin{tabular}{|c|c|c|c|}
\hline $\begin{array}{l}\text { Appartenance } \\
\text { institutionnelle }\end{array}$ & $\begin{array}{c}\text { Nombre de structures } \\
\text { de diagnostic } \\
\text { de TPM+ (\%) }\end{array}$ & $\begin{array}{c}\text { Nombre total } \\
\text { de TPM+ diagnostiqués } \\
\text { en } 2006 \text { (\% du total) }\end{array}$ & $\begin{array}{c}\text { Nombre médian } \\
\text { de TPM+ diagnostiqués par mois } \\
\text { par structure [IC à } 95 \% \text { ] }\end{array}$ \\
\hline Gouvernement & $4 / 15(26,7)$ & $546(54)$ & $4,9[-2,5-12,3]$ \\
\hline Eglise catholique & $5 / 23(21,7)$ & $320(32)$ & $5,4[0,4-10,4]$ \\
\hline Autres Églises & $1 / 8(12,5)$ & $126(13)$ & $10,5[10,5-10,5]$ \\
\hline ONG & $0 / 5(0,0)$ & - & - \\
\hline Entreprises & $0 / 4(0,0)$ & - & - \\
\hline Indépendants & $1 / 164(0,6)$ & $5(1)$ & $0,4[0,4-0,4]$ \\
\hline TOTAL & $11 / 219(5,0)$ & $997(100)$ & - \\
\hline
\end{tabular}

\section{Activités propres aux structures de première ligne}

\section{Vaccination}

Quasi tous les prestataires de la première ligne vaccinent. En 2006, 29370 enfants de moins d'un an ont reçu leur $3^{\text {ième }}$ dose du vaccin DTC dans ces structures. Le taux de couverture en DTC3 est de 49\% (29 370 enfants vaccinés/60 000 enfants attendus).

Le Tableau 6 montre dans quelle mesure ces structures, groupées par appartenance institutionnelle, ont contribué à ce résultat. Il compare aussi les structures en fonction du nombre médian de vaccinations DTC3 effectuées. On constate que les différences observées entre les structures gouvernementales ou catholiques d'une part, et les structures privées indépendantes d'autre part sont statistiquement significatives : ces dernières vaccinent moins.

\section{Diagnostic de la tuberculose pulmonaire à microscopie positive}

Comme indiqué au Tableau 7, le diagnostic de la tuberculose est une activité intégrée dans seulement
11 des 219 structures de soins de première ligne (soit $5 \%$ du total). Ce sont essentiellement des structures gouvernementales et confessionnelles qui pratiquent cette activité. Avec une incidence nationale de TPM+ d'au moins 150 cas pour 100000 habitants (Tableau 1), on devrait s'attendre à dépister au moins 2250 cas à Lubumbashi en 2006 pour une population de 1500000 habitants. En dépistant 997 cas TPM+, le taux de dépistage est de 44,3\% (997/2 250).

\section{Activités propres aux structures intermédiaires et hôpitaux}

\section{Hospitalisations}

En 2006, 58488 malades ont été hospitalisés, donnant un taux d'hospitalisation d'environ 39 hospitalisés pour 1000 habitants. Le Tableau 8 présente les indicateurs classiques permettant d'apprécier l'utilisation des structures hospitalières. La durée moyenne de séjour est nettement plus faible pour les structures intermédiaires que pour les hôpitaux. 
Tableau 8. Durée moyenne de séjour (DMS), Taux d'occupation moyen de lits (TOM) et nombre médian de lits par type de structures, en 2006

\begin{tabular}{|c|c|c|c|c|c|c|c|}
\hline & $\begin{array}{l}\text { Nombre de } \\
\text { structures qui } \\
\text { hospitalisent } \\
\text { (\% du total) }\end{array}$ & $\begin{array}{c}\text { Nombre } \\
\text { de lits } \\
\text { effectifs* } \\
(\% \text { du total) }\end{array}$ & $\begin{array}{l}\text { Nombre } \\
\text { de sorties* }\end{array}$ & $\begin{array}{c}\text { Nombre } \\
\text { de journées } \\
\text { d'hospitalisation* }\end{array}$ & $\begin{array}{c}\text { DMS } \\
\text { (jours) }\end{array}$ & $\begin{array}{c}\text { TOM } \\
(\%)\end{array}$ & $\begin{array}{c}\text { Nombre médian } \\
\text { de lits par type } \\
\text { de structure } \\
\text { [IC à } 95 \%]\end{array}$ \\
\hline $\begin{array}{l}\text { Structures } \\
\text { intermédiaires }\end{array}$ & $23(71,9)$ & $448(23,4)$ & 19958 & 93691 & 4,7 & 57,3 & $16[11,2-20,8]$ \\
\hline Hôpitaux & $9(28,1)$ & $1,465(76,6)$ & 38530 & 267526 & 6,9 & 50,0 & $120[43,6-196,4]$ \\
\hline TOTAL/MOYENNE & E $32(100)$ & $1,913(100)$ & 58488 & 361217 & 6,2 & 51,7 & - \\
\hline
\end{tabular}

* Ces données ne concernent pas les accouchements

Tableau 9. Nombre d'interventions chirurgicales réalisées dans les structures intermédiaires et les hôpitaux, en 2006

\begin{tabular}{|c|c|c|}
\hline & $\begin{array}{cc}\text { Nombre } & \text { Nombre } \\
\text { d'opérés d'hospitalisés } & \text { d'o } \\
\text { (\% du total) } & \text { (\% du total) }\end{array}$ & $\begin{array}{c}\text { Nombre } \\
\text { d'opérés/ } \\
100 \text { malades } \\
\text { hospitalisés }\end{array}$ \\
\hline $\begin{array}{l}\text { Structures } \\
\text { intermédiaires }\end{array}$ & $2140(28,5) 19958(34,1)$ & 11 \\
\hline Hôpitaux & $5360(71,5) 38530(65,9)$ & 14 \\
\hline $\begin{array}{l}\text { TOTAL/ } \\
\text { MOYENNE }\end{array}$ & $7500(100) 58488(100)$ & 13 \\
\hline
\end{tabular}

\section{La chirurgie majeure}

Le Tableau 9 détaille par niveau et par type de structure les 7500 interventions chirurgicales majeures pratiquées au cours de l'année 2006. Plus d'un quart $(28,5 \%)$ ont eu lieu dans des structures intermédiaires et le reste dans des formations hospitalières. En outre, sur 100 malades hospitalisés, 11 subissent une intervention chirurgicale lorsqu'il s'agit d'une structure intermédiaire contre 14 lorsqu'il s'agit d'un hôpital.

\section{Discussion}

Cette étude comporte au moins quatre faiblesses. La première concerne la source des données. En effet, comme le souligne aussi l'OMS (11), les registres administratifs de prestation des services de santé courants utilisés ici comme source de données ont tendance à surestimer la couverture en raison d'erreurs : double comptage affectant le numérateur et incertitudes entachant le dénominateur. La deuxième faiblesse est qu'en visant à analyser dans sa globalité l'offre des soins dans la ville, l'analyse des données par zone de santé ou par structure de soins qui pouvait être pertinente à certains égards échappe à cette étude. La troisième faiblesse est due au fait que les données d'une seule année ne permettent pas de renforcer l'argumentation par une analyse des tendances. La quatrième enfin tient au fait qu'on considère dans cette étude que toutes les consultations curatives sont de premier contact. Ces données ont cependant le mérite de complétude des informations recherchées et d'exhaustivité des cas pour l'année considérée. Les indicateurs qui en découlent permettent de savoir dans quelle mesure les interventions sanitaires importantes bénéficient effectivement aux personnes qui en ont besoin (11) et d'identifier les principaux pôles de recours des patients par rapport aux différents niveaux de soins et aux différents prestataires institutionnels.

\section{Consultations curatives primaires et accouchements}

Le taux d'utilisation des consultations curatives en 2006 est de 0,37 NC/habitant/an (Tableau 2). Ce taux est inférieur à celui préconisé par le ministère de la santé de la RD Congo (voir Tableau 1). Il ne permet sans doute pas de répondre à l'ensemble des problèmes de santé auxquels la population est confrontée (voir l'introduction de ce travail). Il suggère aussi des problèmes d'acceptabilité du secteur formel comme d'accessibilité financière, surtout dans un climat de marchandisation envahissante des soins suite à une dérégulation du système de santé $(1,2,15)$. L'existence d'alternatives comme l'automédication, l'utilisation de prestataires privés informels, voire clandestins, le recours à la médecine traditionnelle, ou encore l'utilisation d'une " médecine " basée sur la prière, la divination, la magie, etc. (6,16-18),

IUHPE - Global Health Promotion Vol.17, No. 32010 
explique sans doute aussi cette faible utilisation des structures formelles. Le recours aux soins et le parcours thérapeutique des malades à Lubumbashi mériteraient une analyse approfondie dans le cadre de l'appréciation de la qualité de soins.

Par ailleurs, le gros des consultations curatives est réalisé par les structures appartenant aux privés indépendants (c'est-à-dire des personnes physiques travaillant en solo ou en association) et ces structures sont aussi les plus nombreuses. Mais, quelle que soit l'appartenance institutionnelle des structures de première ligne, le volume de consultations curatives par jour et par structure reste très faible (Tableau 3). Dans ces conditions, l'acquisition continue et le maintien de compétences par le personnel soignant sont quasi impossibles, surtout quand certaines structures se caractérisent par une pléthore en personnel. Toutefois, dans les structures de soins de première ligne d'entreprises, le volume d'activités est nettement plus élevé et l'affectation du personnel y serait plus rationnelle. Nous faisons l'hypothèse que la fréquentation élevée des structures d'entreprises est la conséquence de la gratuité des soins pour les employés couverts et leurs familles, et de l'adaptation du nombre de structures au volume de travail attendu. Cette analyse suggérerait aussi qu'une meilleure accessibilité financière des autres structures de soins augmenterait le nombre de consultations curatives.

Le taux d'accouchements assistés à Lubumbashi a été évalué à $86,9 \%$. Ce taux est une approximation, les naissances attendues dérivées du taux brut de natalité ayant pris en compte les mort-nés. Ce chiffre est comparable à la couverture moyenne des accouchements assistés en milieu urbain congolais (de $83 \%$ en 2001 à $91 \%$ en 2007$)(19,20)$. Il se situe dans les limites de l'objectif national qui est d'au moins $80 \%$. Il reste aussi dans les limites des taux d'accouchements assistés (62-98\%) observés dans les capitales et d'autres grandes villes des pays d'Afrique centrale (20-23). Le nombre relativement important des structures de soins avec maternités, ainsi qu'une forte sensibilisation de la population par le programme de santé de la reproduction, peuvent contribuer à expliquer ce résultat. Une autre explication est la plus grande souplesse dans la délivrance des déclarations de naissances à partir de la maternité grâce à une attitude plus flexible des services municipaux. L'idéal serait que toutes les femmes enceintes accouchent en présence d'un personnel de santé qualifié, des efforts restent donc à fournir pour se rapprocher de cet idéal.

Cependant, si la césarienne est pratiquée dans toutes les structures intermédiaires et hospitalières de Lubumbashi (Tableau 4), son taux $(1,8 \%$ des accouchements) reste très en dessous de la fourchette de $5-15 \%$ qui est l'intervalle de variation de taux de césariennes nécessaires pour éviter la morbidité et la mortalité maternelle et néonatale $(14,24)$. Ceci laisse penser qu'une partie importante de besoins obstétricaux ne sont pas couverts (24) dans un environnement où les moyens techniques modernes de surveillance materno-fœetale font cruellement défaut. Une étude portant sur l'évolution de la fréquence des césariennes et de la mortalité materno-néonatale à la maternité de l'hôpital Sendwe, la plus grande formation médicale de Lubumbashi (7), a montré que, sur une période de 5 ans, la fréquence des césariennes n'a jamais dépassé $2 \%$ et que la mortalité maternonéonatale est plus liée aux tentatives indues et infructueuses d'accoucher par voie basse. Ce taux reste aussi inférieur à ceux rapportés dans d'autres villes des pays d'Afrique centrale (20-23) et d'Afrique sub-saharienne en général (25).

En définitive, en matière de consultations curatives primaires et d'accouchements par voie basse, l'hôpital est bien plus sollicité que les structures intermédiaires ou de première ligne, et les raisons ne sont pas encore connues et mériteraient d'être investiguées. Il se peut que la perception de la qualité des soins au niveau de la $1^{\text {ère }}$ ligne soit problématique, ou encore que le " court-circuitage » de la première ligne cadre avec un système de compétition franche entre les différents niveaux de soins au niveau de la zone de santé. Il est vraisemblable qu'une grande partie de consultations et d'accouchements qui ont lieu à l'hôpital, suite à l'accès direct des malades et des parturientes à cet échelon de soins, aurait pu prendre place au niveau d'un service de santé de première ligne fonctionnel et aisément accessible à la population.

\section{Vaccination et diagnostic de la tuberculose}

Dans notre étude, nous notons que $67,6 \%$ des structures de première ligne vaccinent (Tableau 6). Ceci procède de la stratégie de décentralisation avec intégration de toutes les activités de vaccination dans des structures de base, même privées, afin de maximiser la couverture vaccinale. Le fait que presque $2 / 3$ des structures de première ligne qui vaccinent 
appartiennent aux privés indépendants (Tableau 6) doit être vu comme une adaptation de leur offre de soins à la demande des autorités sanitaires de zones ; la vaccination des jeunes enfants étant devenue un axe prioritaire de santé publique. Malgré cela, le taux de couverture vaccinale de $49 \%$ pour le DTC3 demeure de loin inférieur à l'objectif national qui est d'au moins $90 \%$ pour contrôler ou éliminer les maladies prévenues par ce vaccin. Cela dénote un taux d'abandon très élevé et la nécessité opérationnelle de stratégies de récupération (échéancier, visites à domicile...) et la sensibilisation des parents pour améliorer la situation pour ce vaccin à trois doses.

Pour la tuberculose, $5 \%$ seulement des structures de première ligne font le diagnostic de TPM+. Ceci tient à l'organisation même de la lutte contre la tuberculose au niveau opérationnel qui prône une décentralisation partielle et l'intégration de quelques activités de lutte ne nécessitant pas de techniques et compétences spécifiques (26). C'est plutôt pour répondre aux exigences - tout à fait rationnelles, mais sélectives - de la politique en la matière que du manque d'intérêt des prestataires à intégrer les activités de diagnostic de TPM+ dans leurs structures qu'il faut expliquer la faible couverture de ces activités dans la ville. Le taux de dépistage est de $44,3 \%$. Il est loin de l'objectif national, d'au moins $70 \%$, nécessaire pour interrompre la chaîne de transmission. Un équilibre devrait être trouvé entre la qualité de ser vices recherchée et le nombre de structures de dépistage de la tuberculose manifestement insuffisant.

\section{Hospitalisation et chirurgie majeure}

Concernant l'offre de soins hospitaliers à Lubumbashi, l'Etat reste le prestataire majoritaire en fournissant plus de la moitié des lits d'hospitalisation disponibles (1). Ensemble, les structures d'hospitalisation de Lubumbashi offrent 1913 lits soit 13 lits/10,000 habitants. Le taux d'hospitalisation et le taux d'occupation de lits sont relativement bas : respectivement 39 pour 1000 habitants par an et $51,7 \%$. En comparant avec les autres villes, on note qu'il n'y a pas pléthore de lits et donc l'hypothèse explicative la plus probable du faible TOM est celle d'une sous-utilisation due à un problème d'accessibilité des soins hospitaliers. Une durée moyenne de séjour d'environ 6 jours est comparable à ce qu'on observe dans d'autres hôpitaux du pays et de la région (27 28). La durée moyenne de séjour plus courte dans les structures intermédiaires suggère que les patients hospitalisés dans ces services le sont pour des problèmes moins sévères.

L'étude révèle que $13 \%$ des malades hospitalisés à Lubumbashi subissent une intervention chirurgicale majeure et que plus de $1 / 4$ de ces interventions se déroulent dans des structures intermédiaires. Lorsqu'on considère les conditions de travail parfois très précaires observées dans certaines de ces structures lors de notre enquête, il y a lieu de s'interroger sur l'efficacité de ces interventions sur la santé du malade, voire même sur sa sécurité physique. Ceci mériterait d'être étudié plus en détail.

\section{Conclusion}

Cet article fait une analyse critique des principales activités de santé réalisées dans les structures de soins formelles dans la ville de Lubumbashi. Des consultations curatives sont offertes dans presque toutes les structures de soins, tous niveaux et appartenances institutionnelles confondus. L'hôpital est le principal pôle d'attraction des patients. Cependant, le taux d'utilisation des consultations curatives reste relativement faible au regard des problèmes réels de santé de la population. Pour mieux comprendre cet état des choses, il serait intéressant de mener une étude sur les itinéraires thérapeutiques des malades couplée à une étude sur l'accessibilité financière et la qualité des soins. Il conviendrait d'encourager davantage l'accouchement en présence d'un personnel de santé qualifié. La césarienne devrait être promue dans ses indications justifiées. Quant aux services de vaccination, le faible taux d'enfants ayant reçu leur $3^{\text {ème }}$ dose de DTC est une question qui mérite une attention particulière. L'implication des prestataires privés dans la vaccination pourrait être considérée comme une prédisposition au dialogue avec l'Etat dans une perspective de contractualisation pour une couverture universelle. Cette étude a aussi révélé une discordance entre la disponibilité des services de diagnostic des TPM+ et l'ampleur du problème dans la ville. Ceci justifie une augmentation conséquente des centres de diagnostic pour rencontrer les besoins réels de la population dans ce domaine.

Il serait intéressant de conduire une étude similaire à celle décrite dans ce travail quelques années après le démarrage effectif de la SRSS afin d'apprécier l'impact de cette stratégie. 


\section{Notes}

i. Cette taille de la population est l'estimation la plus réaliste au regard des chiffres très divergents utilisés d'une part par les services de l'Etat civil et d'autre part par les services de santé.

ii. Appendicectomie, cure de hernie, diverses laparotomies (grossesse ectopique, myome...).

\section{Références}

1. Chenge MK, Van der Vennet J, Porignon D, Luboya NO, Kabyla I, Criel B. La carte sanitaire de la ville de Lubumbashi, République Démocratique du Congo. Partie I : problématique de la couverture sanitaire en milieu urbain congolais. Glob Health Promot. 2010; 17(3).

2. Ministère de la santé. Stratégie de renforcement du système de santé. Kinshasa, République Démocratique du Congo; 2006.

3. Grodos D. Le district sanitaire urbain en Afrique subsaharienne : enjeux, pratiques et politiques. Louvain-laNeuve : Université catholique de Louvain : département de santé publique, Faculté de médecine ; 2000.

4. Harpham T, Burton S, Blue I. Healthy city projects in developing countries : the first evaluation. Health Promot Int. 2001; 16: 111-25.

5. Harpham T. Urban health in developing countries : what do we know and where do we go? Health Place. 2009; 15: 107-16.

6. Kakoma S. Le profil sanitaire du Lushois. Rapport des recherches effectuées durant la troisième session des travaux de l'observatoire du changement urbain, février-juin 2001. Lubumbashi : Presses universitaires de Lubumbashi; 2002.

7. Kizonde K, Kinenkinda X, Kimbala J, Kamwenyi K. La césarienne en milieu africain : exemple de la maternité centrale Sendwe de Lubumbashi, RD Congo. Méd Afr Noire. 2006; 53: 293-8.

8. Criel B, Macq J, Bossyns P, Hongoro C. A coverage plan for health centres in Murewa district in Zimbabwe : an example of action research. Trop Med Int Health. 1996; 1: 699-709.

9. Doherty J, Rispel L, Webb N. Developing a plan for primary health care facilities in Soweto, South Africa. Part II : applying locational criteria. Health Policy Plan. 1996; 11: 394-405.

10. Samake S, Dakono G, Guidetti F. La carte sanitaire : outil de négociation pour le développement sanitaire? In : Brunet-Jailly E, éditeur, Innover dans les systèmes de santé : expériences d'Afrique de l'Ouest. Paris : Editions Karthala; 1997.

11. OMS. Statistiques sanitaires mondiales; 2009.

12. Van Lerberghe W, Van Balen H, Kegels G. Typologie et performances des hôpitaux de premier recours en Afrique sub-saharienne. Annales de la Société belge de médecine tropicale. 1992; 72: 1-51.

13. Van Lerberghe W, Lafort Y. Le rôle de l'hôpital dans le district : dispenser ou soutenir les soins de santé primaires ? Organisation Mondiale de la Santé. Division de Renforcement des Services de Santé. WHO/SHS/CC/ 90.2 Mimeo; 1990.

14. D’Altilia J-P, De Lamalle J-P, De Caluwé P, Greindl I, Lecherlier F, Wodon A. Système d'information sanitaire; 2005.

15. OMS. Les soins de santé primaires - Maintenant plus que jamais. Rapport sur la santé dans le monde 2008; 2009.

16. Develay A, Sauerborn R, Diesfeld H. Utilization of health care in an African urban area: results from a household survey in Ouagadougou, Burkina-Faso. Soc Sci Med. 1996; 43: 1611-9.

17. Harpham T, Molyneux C. Urban health in developing countries: a review. Progress in Development Studies. 2001; 1: 113-37.

18. Dugas S, Van Dormael M. La construction de la médecine de famille dans les pays en développement. Studies in Health Services Organisation \& Policy. 2003; 22: 1-352.

19. Ministère du plan et de la reconstruction. Enquête nationale sur la situation des enfants et des femmes MICS2/2001. Rapport d'analyse; 2002. Kinshasa, République Démocratique du Congo.

20. Ministère du Plan et Macro International. Enquête Démographique et de Santé, République Démocratique du Congo 2007. Calverton, MD: Ministère du Plan et Macro International; 2008.

21. Central Statistical Office, Ministry of Health, Tropical Diseases Research Centre TDRC, University of Zambia and Macro International Inc. Zambia Demographic and Health Survey 2007. Calverton, MD : CSO and Macro International; 2009.

22. Centre National de la Statistique et des Études Économiques (CNSEE) et ORC Macro. Enquête Démographique et de Santé du Congo 2005. Calverton, MD : CNSEE et ORC Macro, 2006.

23. Uganda Bureau of Statistics (UBOS) and Macro International Inc. Uganda Demographic and Health Survey 2006. Calverton, MD: UBOS and Macro International; 2007.

24. De Brouwere V, Van Lerberghe W. Les besoins obstétricaux non couverts. Paris : L'Harmatan; 1998.

25. Buekens P, Curtis S, Alayon S. Demographic and Health Surveys: caesarean section rates in sub-Saharan Africa. BMJ. 2003; 326: 136.

26. Ministère de la santé/Programme national de lutte contre la tuberculose. Guide de prise en charge de la tuberculose PATI 4. Kinshasa; 2008.

27. Atkinson S, Ngwengwe A, Macwan'gi M, Ngulube TJ, Harpham T, O'Connell A. The referral process and urban health care in sub-Saharan Africa: the case of Lusaka, Zambia. Soc Sci Med. 1999; 49: 27-38.

28. Ekwanzala M, Ngo B, Moudi A. La réhabilitation intégrale : une approche pour la relance du système de santé en République Démocratique du Congo. Kinshasa : Centre Protestant d'Edition et de Diffusion; 2001. 